\title{
Designing Meta Material Slabs Exhibiting Negative Refraction Using Topology Optimization
}

Christiansen, Rasmus Ellebæk; Sigmund, 0.

Published in:

Structural and Multidisciplinary Optimization

Link to article, DOI:

$10.1007 / \mathrm{s} 00158-016-1411-8$

Publication date:

2016

Document Version

Peer reviewed version

Link back to DTU Orbit

Citation (APA):

Christiansen, R. E., \& Sigmund, O. (2016). Designing Meta Material Slabs Exhibiting Negative Refraction Using Topology Optimization. Structural and Multidisciplinary Optimization, 54(3), 469-482.

https://doi.org/10.1007/s00158-016-1411-8

\section{General rights}

Copyright and moral rights for the publications made accessible in the public portal are retained by the authors and/or other copyright owners and it is a condition of accessing publications that users recognise and abide by the legal requirements associated with these rights.

- Users may download and print one copy of any publication from the public portal for the purpose of private study or research.

- You may not further distribute the material or use it for any profit-making activity or commercial gain

- You may freely distribute the URL identifying the publication in the public portal

If you believe that this document breaches copyright please contact us providing details, and we will remove access to the work immediately and investigate your claim 


\title{
Designing Meta Material Slabs Exhibiting Negative Refraction Using Topology Optimization
}

\author{
Rasmus E. Christiansen, Ole Sigmund.
}

Received: date / Accepted: date

\begin{abstract}
This paper proposes a topology optimization based approach for designing meta materials exhibiting a desired negative refraction with high transmission at a given angle of incidence and frequency. The approach considers a finite slab of meta material consisting of axis-symmetric designable unit cells subjected to an exterior field. The unit cell is designed to achieve the desired properties based on tailoring the response of the meta material slab under the exterior field. The approach is directly applicable to physical problems modeled by the Helmholtz equation, such as acoustic, elastic and electromagnetic wave problems. Acoustic meta materials with unit cell size on the order of half the wave length are considered as examples. Optimized designs are presented and their performance under varying frequency and angle of incidence is investigated.
\end{abstract}

Keywords topology optimization - negative refraction · wave propagation - meta materials - acoustics . electromagnetics $\cdot$ elastics

\section{Introduction}

In this paper the negative refraction of waves at a material interface is understood from Snell's law and the refractive index, $n$, is defined accordingly. This is not identical to the definition $n=c / c_{p}$, where $c$ is the wave speed in vacuum and $c_{p}$ is the wave phase velocity in the material.

Materials exhibiting negative refraction of acoustic, elastic or electromagnetic waves have been of great interest over the last decades, see e.g. (Krowne and Zhang, 2007, Craster

R. E. Christiansen $(*) \cdot$ O. Sigmund

Department of Mechanical Engineering, Solid Mechanics,

Technical University of Denmark, Nils Koppels Alle, B. 404

DK-2800 Lyngby, Denmark

E-mail: (*) raelch@ mek.dtu.dk and Guenneau, 2013) for an introduction to and overview of the topic. The work by Pendry (2000) discussing the application of materials exhibiting negative refraction in designing a perfect optical lens was instrumental in sparking the interest in negative refraction. Another reason for the interest is that a material exhibiting this behavior may simultaneously have negative material parameters, as first discussed theoretically for the electromagnetic case by Veselago (1968). Having access to materials exhibiting negative refraction and to materials with negative material parameters, and even better being able to fine-tune the behavior of such materials, opens up a range of new possibilities in science and engineering by offering a new way of manipulating fields. The most studied example being improvements in focusing and lensing, see e.g. (Mills et al, 2003; Xu et al, 2013; Zhang et al, 2009).

Materials exhibiting negative refraction and/or having negative material parameters are not readily available in nature however. Therefore so called meta materials (MMs) consisting of an array of unit cells with the desired properties must be created artificially through careful design. A vast amount of research has gone into understanding and designing MMs with negative material parameters and MMs exhibiting negative refraction, see e.g. (Zhang and Liu, 2004; Zhang et al, 2005, Philippe et al 2015) for examples for acoustic, electromagnetic and elastic waves respectively.

In most previous work the design of the MMs has relied on a range of simplifying assumptions for the model problem used to model the physics under consideration, such as: Homogenization techniques where effective parameters for the MM can be extracted from a given unit cell design, which are only strictly valid when the unit cell size is much smaller than the wave length; Considering a single unit cell 
with the assumptions of an infinitely periodic MM hereby neglecting any edge effects at interfaces; Considering band diagrams and identifying a $(k, \omega)$-range with negative slope under the assumption that only a single mode is excited inside the MM, hereby disregarding more complex wave interactions inside the MM.

In this work a novel approach to the problem of designing meta material slabs exhibiting negative refraction is suggested. The approach is based on applying topology optimization, (Bendsøe and Sigmund, 2003), to determine the material distribution inside the unit cells of the MM in order to achieve the desired property. Whereas most other publications on the topic have assumed either infinite periodicity or have based their meta material design on the evaluation of transmission studies through just one layer of unit cells, we here base our designs on finite slabs with arbitrary numbers of layers and model the full wave field impinging on the slab. In this way both edge effects of the slab as well as the complete field inside the MM are automatically accounted for in the design process. When applied successfully, the proposed approach allows for the design of MM slabs exhibiting a desired negative refraction to within $\mathrm{a} \approx 10 \%-20 \%$ margin of error with a transmittance through the full slab of more than $95 \%$ for a given incoming angle and frequency. Previous work applying topology optimization as a tool for designing MMs exhibiting effective negative parameters include, (Diaz and Sigmund, 2009, Zhou et al, 2010, Lu et al, 2013). Other interesting recent applications of topology optimization for designing MMs with exotic behavior include the work by Park et al (2015) on self collimating phononic crystals and the work by Andkjær and Sigmund (2013) on designing cloaks for acoustics and electromagnetics.

Although this paper presents examples for two dimensional acoustics the approach has also successfully been tested for designing MMs exhibiting negative refraction for both TE and TM polarized electromagnetic waves and shear waves in solids, in two dimensions. During the testing it was found that problems with high contrast in material parameters, such as the acoustic case treated here, are the most challenging to treat. Except for computational limitations, the method is not restricted to two dimensions.

\section{The Model Problem}

Consider an exterior wave problem governed by the Helmholtz equation (1), defined on the domain, $\Omega \subset \mathbb{R}^{2}$, illustrated in Fig. 1

$\boldsymbol{\Omega}$ is truncated by the curve $\delta \boldsymbol{\Omega}$, along which a far field matching condition is imposed to fulfill the Sommerfeld

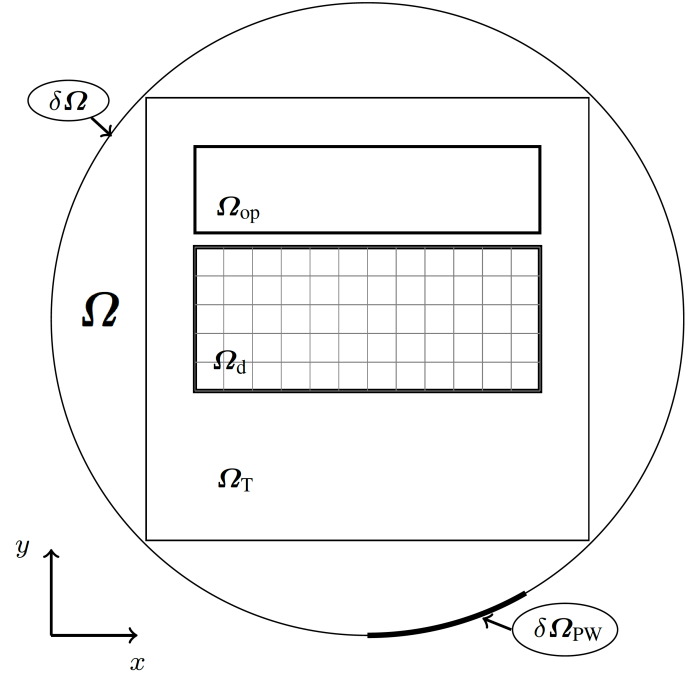

Fig. 1: Model problem sketch. $\boldsymbol{\Omega}$ : Truncated Domain. $\boldsymbol{\Omega}_{\mathrm{op}}$ : Optimization domain. $\boldsymbol{\Omega}_{\mathrm{d}}$ : Design domain. $\delta \boldsymbol{\Omega}$ : Truncated Domain boundary. $\delta \Omega_{\mathrm{PW}}$ : Excitation boundary.

radiation condition (2) corresponding to perfectly absorbing boundary conditions. A boundary condition (3) is imposed along a section of $\delta \Omega$, denoted $\delta \Omega_{\mathrm{PW}}$ in order to excite a localized plane wave entering and traveling through $\Omega$. For use in the optimization problem two subdomains, $\boldsymbol{\Omega}_{\mathrm{d}}$ and $\Omega_{\mathrm{OP}}$ are defined. $\boldsymbol{\Omega}_{\mathrm{d}}$ denotes the design domain and is where the designable MM slab consisting of an array of axis symmetric unit cells is placed. $\Omega_{\mathrm{OP}}$ denotes the optimization domain and is where the objective function for the optimization problem is defined. $\Omega_{\mathrm{T}}$ denotes the truncated modeling domain used when displaying the solution field and design. The model problem can be written as

$$
\begin{aligned}
\nabla \cdot\left(\frac{1}{\alpha(\mathbf{x})} \nabla \psi(\mathbf{x})\right)+\omega^{2} \frac{1}{\gamma(\mathbf{x})} \psi(\mathbf{x}) & =0, \quad \mathbf{x} \in \boldsymbol{\Omega}, \\
\lim _{|\mathbf{r}| \rightarrow \infty} \sqrt{|\mathbf{r}|} \cdot\left(\frac{\partial \psi(\mathbf{r})}{\partial|\mathbf{r}|}+\mathrm{i} \omega \cdot \sqrt{\frac{\alpha}{\gamma}} \psi(\mathbf{r})\right) & =0, \quad \mathbf{x} \in \delta \boldsymbol{\Omega} \\
\mathbf{n} \cdot\left(\frac{1}{\alpha(\mathbf{x})} \nabla \psi\right) & =a_{n}, \mathbf{x} \in \delta \boldsymbol{\Omega}_{\mathrm{PW}} .
\end{aligned}
$$

In equations (1)-(3) $\mathbf{x}$ and $\mathbf{r}$ denote the spatial dependence in Cartesian and polar coordinates respectively, $\mathrm{i}$ is the imaginary unit, $\psi$ is the field of interest, $\omega=2 \pi f$ is the angular frequency with $f$ being the frequency. $\alpha(\mathbf{x})$ and $\gamma(\mathbf{x})$ are material parameters and depend on the material at the point $\mathbf{x}$. Finally $a_{n}$ is calculated by prescribing a known $\psi$ on $\delta \Omega_{\mathrm{PW}}$ using the following expression describing a Gaussian shaped plane wave 


$$
\begin{aligned}
\psi_{\mathrm{GPW}} & =\mathrm{e}^{-\left(\frac{\left(\mathbf{x}-\mathbf{x}_{w}\right) \cdot \hat{\mathbf{d}}}{\delta_{x}}\right)^{2}} \mathrm{e}^{-\mathrm{i} \hat{\omega}(\mathbf{x} \cdot \mathbf{d})}, \\
\mathbf{d} & =\left(\begin{array}{c}
\cos (\pi / 2+\theta) \\
\sin (\pi / 2+\theta)
\end{array}\right), \quad \mathbf{x}_{w}=\left(\begin{array}{l}
x_{w} \\
y_{w}
\end{array}\right) .
\end{aligned}
$$

Here $\delta_{x}$ controls the width of the Gaussian envelope, $\mathbf{d}$ controls the propagation direction of the wave, $\hat{\mathbf{d}}$ denotes the transpose direction vector, finally $\mathbf{x}_{w}$ controls the position of the center of the wave. Henceforth the spatial dependence of quantities is suppressed for brevity, except when needed. See Fig. 2 for a simplified sketch of the solution of (11)-(3) superimposed on $\Omega$ in light gray, given the imposition of (4) on $\delta \boldsymbol{\Omega}_{\mathrm{PW}}$ with an empty $\boldsymbol{\Omega}_{\mathrm{d}}$. Note that $\theta=\theta_{1}$ is measured positively as illustrated in Fig. 2

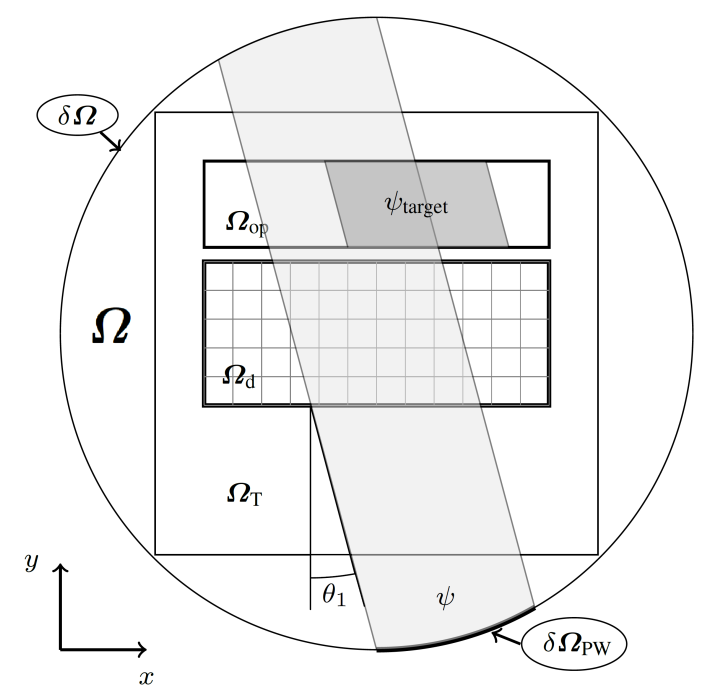

Fig. 2: $\psi$ : Enveloped plane wave (Solution to (1)-(3) and $\psi_{\text {target }}$ : Target wave, overlaid on modeling domain. $\Omega$ : Truncated Domain. $\boldsymbol{\Omega}_{\mathrm{op}}$ : Optimization domain. $\boldsymbol{\Omega}_{\mathrm{d}}$ : Design domain. $\delta \boldsymbol{\Omega}:$ Truncated Domain boundary. $\delta \Omega_{\mathrm{PW}}$ : Excitation boundary.

For the examples treated in this paper the model problem is non-dimensionalized and rescaled as

$\hat{\alpha}=\left\{\begin{array}{ll}1 & \text { void } \\ \frac{\alpha_{\text {solid }}}{\alpha_{\text {void }}} & \text { solid }\end{array}, \hat{\gamma}=\left\{\begin{array}{ll}1 & \text { void } \\ \frac{\gamma \text { solid }}{\gamma \text { void }} & \text { solid }\end{array}, \hat{\omega}=\frac{\omega}{c}\right.\right.$.

Here $c \equiv 1$ is a normalized and non-dimensional wave speed. For the acoustic problem treated in the paper, $\psi$ is the sound pressure while $\alpha=\rho$ and $\gamma=\kappa$ are the density and the bulk modulus, respectively. Each position in space may either consist of a solid perfectly reflecting material or void (air). For the elastic case, $\psi$ is the displacement field while $\alpha$ and $\gamma$ relate to the density and shear modulus. For the transverse electromagnetic cases $\psi$ is either the electric or magnetic field while $\alpha$ and $\gamma$ relate to the electric permeability and permittivity.

\subsection{Discretization}

For the simulation results presented in this work the model problem is discretized using a hybrid wave based method and a finite element method (hybrid WBMFEM method), developed for acoustic problems modeled using the Helmholtz equation in 2D and 3D, (Desmet, 1998; Pluymers 2006). The method has previously been applied for topology optimization by Goo et al (2014) and Christiansen et al (2015b). The design domain, $\boldsymbol{\Omega}_{\mathrm{d}}$, is discretized using $\mathcal{N}_{e}$ bi-linear finite elements and the rest of the domain, $\boldsymbol{\Omega} \backslash \boldsymbol{\Omega}_{\mathrm{d}}$ is discretized using the wave based method. The two disjoint subdomains are coupled using an indirect impedance coupling described in chapter 7 of (Pluymers, 2006). The far field boundary condition is implemented using the method proposed in chapter 6 of (Pluymers, 2006). Although the hybrid WBM-FEM method is more complex to implement than a pure FEM method and has certain restrictions, it has the strength that very few degrees of freedom are needed in the WBM part of the domain. This significantly reduces the size of the linear system to be solved to obtain $\psi$ hereby saving significant computational resources.

\section{The Optimization Problem}

The proposed approach applies topology optimization as the tool for designing the meta material slabs and consists of formulating and solving a constrained optimization problem. The objective of the problem is to minimize the amplitude difference between a target enveloped plane wave, $\psi_{\text {target }}$ (illustrated in Fig. 2 using dark gray), and $\psi$, the solution of (1)-(3) over $\Omega_{\mathrm{OP}}$. This difference is minimized by introducing a periodic array of axis-symmetric identical unit cells in $\Omega_{\mathrm{d}}$ (the MM slab). The chosen $\psi_{\text {target }}$ takes the form given in (4) with identical direction, $\mathbf{d}$, and width, $\delta_{x_{\text {target }},}$ as the wave generated by the imposed boundary condition on $\delta \Omega_{\mathrm{PW}}$. The position of the center of $\psi_{\text {target }}$, $\mathbf{x}_{w_{\text {target }}}$, is chosen based on the desired refractive index, $n$ for a given $\theta_{1}$ using (22) in section 4

The objective function, $\Phi$, to be minimized, is formulated based on the standard deviation, $\operatorname{STD}(x)$, between $|\psi|^{2}$ and $\left|\psi_{\text {target }}\right|^{2}$ and is defined on $\boldsymbol{\Omega}_{\mathrm{OP}}$ as 


$$
\begin{gathered}
\Phi=c_{s} \cdot \underset{\boldsymbol{\Omega}_{\mathrm{OP}}}{\mathbf{S T D}}\left(|\psi|^{2}-\left|\psi_{\text {target }}\right|^{2}\right), \\
\underset{\boldsymbol{\Omega}_{\mathrm{OP}}}{\mathbf{S T D}}(x)=c_{s} \cdot \frac{\int\left(x-\frac{\int x d \boldsymbol{\Omega}_{\mathrm{OP}}}{\int d \boldsymbol{\Omega}_{\mathrm{OP}}}\right)^{2} d \boldsymbol{\Omega}_{\mathrm{OP}}}{\int d \boldsymbol{\Omega}_{\mathrm{OP}}},
\end{gathered}
$$

where $c_{s}$ is a scaling parameter used to adjust the magnitude of $\Phi$ to improve the performance of the optimization algorithm. If $\Phi$ reaches a very low value it is beneficial to modify it by applying the natural logarithm to further improve the performance of the optimization algorithm.

The discrete optimization problem of minimizing $\Phi$ by placing solid material in $\Omega_{\mathrm{d}}$ is replaced by a continuous optimization problem through the introduction of an auxiliary design field, $\xi(\mathbf{x}) \in[0,1] \forall \mathbf{x} \in \boldsymbol{\Omega}_{\mathrm{d}}, \xi(\mathbf{x})=$ $0 \forall \mathbf{x} \in \boldsymbol{\Omega} \backslash \boldsymbol{\Omega}_{\mathrm{d}}$. This field is used to interpolate between the inverse material parameters for solid and void as, $\hat{\alpha}(\xi)^{-1}=\alpha_{\text {void }}^{-1}+\xi\left(\alpha_{\text {solid }}^{-1}-\alpha_{\text {void }}^{-1}\right)$ and $\hat{\gamma}(\xi)^{-1}=\gamma_{\text {void }}^{-1}+$ $\xi\left(\gamma_{\text {solid }}^{-1}-\gamma_{\text {void }}^{-1}\right)$, which allows for a continuous transition between solid and void at a given position in space. This material interpolation agrees with the choice by Dühring et al (2008) and is natural for the acoustic case since the inverse material parameters appear directly in the Helmholtz equation (11). The design field, $\xi$, is discretized into a piecewise constant field leading to a finite number of design variables (one per finite element used to discretize $\Omega_{\mathrm{OP}}$, $\left.\mathcal{N}_{e}\right)$, i.e.

$\xi=\sum_{i \in \mathcal{N}_{e}} \xi_{i}, \quad \xi_{i}= \begin{cases}{[0,1]} & \text { in the } i \text { 'th finite element } \\ 0 & \text { elsewhere }\end{cases}$

the design field of one unit cell is mapped to all the other unit cells. A volume constraint (11) is imposed on the amount of material that may be used in the design and the continuous optimization problem is stated as a min/max problem 10) allowing for optimizing for multiple frequencies and multiple angles of incidence simultaneously.

$$
\begin{aligned}
& \min _{\xi} \max _{\theta_{1}, f}\left(\Phi\left(\psi\left(\mathbf{x}, \xi, \theta_{1}, f\right), \psi_{\text {target }}\left(\mathbf{x}, \theta_{1}, f\right)\right)\right), \\
& \text { s.t. } \left.\left.\frac{1}{A_{\boldsymbol{\Omega}_{\mathrm{d}}}} \int \xi \mathrm{d} \boldsymbol{\Omega}_{\mathrm{d}} \leq V, \quad V \in\right] 0,1\right], A_{\boldsymbol{\Omega}_{\mathrm{d}}}=\int \mathrm{d} \boldsymbol{\Omega}_{\mathrm{d}} .
\end{aligned}
$$

Here $\psi\left(\mathbf{x}, \xi, \theta_{1}, f\right)$ is obtained by solving (17)-(3) for a given realization of $\xi, \theta_{1}$ and $f$ while $\psi_{\text {target }}\left(\mathbf{x}, \theta_{1}, f\right)$ is calculated using (4)-(5).

The optimization problem (10)-(11) is recast to the standard bound-formulation presented in (2.3) in Svanberg (2002) and solved accordingly using The Globally Convergent Method of Moving Asymptotes (GCMMA) (Svanberg, 1987,
2002), by selecting the appropriate constants in the call to GCMMA. A maximum of 3 inner iterations per outer iteration and move limits on $\xi_{i}$ of 0.05 for each outer iteration are used. The gradient information, $\frac{\mathrm{d} \Phi}{\mathrm{d} \xi_{i}}$, needed in the optimization, (12)-(13), is obtained by calculating the sensitivites of $\Phi$ towards changes in $\xi$ using discrete adjoint sensitivity analysis, see Dühring et al (2008) and references therein,

$\frac{\mathrm{d} \Phi}{\mathrm{d} \xi_{i}}=\frac{\partial \Phi}{\partial \xi_{i}}+\Re\left(\boldsymbol{\lambda}^{\mathrm{T}} \frac{\partial \mathbf{S}}{\partial \xi_{i}} \boldsymbol{\psi}\right), \mathbf{S}^{T} \boldsymbol{\lambda}=-\left(\frac{\boldsymbol{\delta} \boldsymbol{\Phi}}{\boldsymbol{\delta} \boldsymbol{\psi}}\right)^{\mathrm{T}}$.

The j'th component of $\left(\frac{\delta \Phi}{\delta \psi}\right)^{\mathrm{T}}$ is given as

$$
\begin{gathered}
\left(\frac{\boldsymbol{\delta} \boldsymbol{\Phi}}{\boldsymbol{\delta} \boldsymbol{\psi}}\right)_{j}=\left(\frac{\partial \Phi}{\partial \psi_{R_{j}}}-\mathrm{i} \frac{\partial \Phi}{\partial \psi_{I_{j}}}\right)=4 \cdot c_{s} \cdot \frac{1}{\int d \boldsymbol{\Omega}_{\mathrm{OP}}} . \\
\int\left(\left(|\psi|^{2}-\left|\psi_{\mathrm{target}}\right|^{2}\right)-\frac{\int\left(|\psi|^{2}-\left|\psi_{\mathrm{target}}\right|^{2}\right) d \boldsymbol{\Omega}_{\mathrm{OP}}}{\int d \boldsymbol{\Omega}_{\mathrm{OP}}}\right) . \\
\left(\phi_{j} \bar{\psi}-\frac{\int\left(\phi_{i} \bar{\psi}\right) d \boldsymbol{\Omega}_{\mathrm{OP}}}{\int d \boldsymbol{\Omega}_{\mathrm{OP}}}\right) d \boldsymbol{\Omega}_{\mathrm{OP}} .
\end{gathered}
$$

In (12)-(13) $S$ is the discretized system matrix for (11)-(3), $\psi$ is the solution vector for the discrete problem $\mathbf{S} \psi=\mathbf{f}$ and $\mathbf{f}$ is introduced by the boundary conditions (2)-(3) as well as the coupling between the FEM and WBM subdomains. $\phi_{j}$ is the $j^{\prime}$ th basis function used to represent the solution, $\psi=\sum_{j} \psi_{j} \phi_{j}$, with $\psi_{j}=\psi_{R_{j}}+\mathbf{j} \psi_{I_{i}}$. Finally ${ }^{-}$denotes the complex conjugate.

To assure that the final design is physically admissible, i.e. consisting purely of solid and void regions, a projection operator along with a smoothing filter is applied to the design field, (Guest et al, 2004; Xu et al, 2010; Wang et al 2011). The projection operator is given as

$\hat{\tilde{\xi}}(\tilde{\xi})=\frac{\tanh (\beta \eta)+\tanh (\beta(\tilde{\xi}-\eta))}{\tanh (\beta \eta)+\tanh (\beta(1-\eta))}$.

where $\eta$ is the projection level and $\beta$ is the projection strength. A continuation scheme for $\beta$ is applied, where $\beta=1$ is used initially and $\beta$ is doubled every 25 iterations or whenever the objective value changes less than $10^{-3}$ of the previous objective value for 5 consecutive iterations. The smoothing filter is given as

$$
\begin{aligned}
& \tilde{\xi}\left(\mathbf{x}_{i}\right)=\frac{\int_{\Omega_{\mathrm{d}}} w\left(\mathbf{x}_{i}-\mathbf{x}_{j}\right) \xi\left(\mathbf{x}_{j}\right) \mathrm{d} \mathbf{x}_{j}}{\int_{\Omega_{\mathrm{d}}} w\left(\mathbf{x}_{i}-\mathbf{x}_{j}\right) \mathrm{d} \mathbf{x}_{j}}, \\
& w(\mathbf{x})= \begin{cases}R-|\mathbf{x}| & \forall|\mathbf{x}| \leq R \wedge \mathbf{x} \in \boldsymbol{\Omega}_{\mathrm{d}} \\
0 & \text { otherwise }\end{cases}
\end{aligned}
$$


where $R$ is the filter radius.

The application of the smoothing and projection on $\xi$ means that instead of interpolating the material parameters, $\alpha^{-1}$ and $\gamma^{-1}$ using $\xi$, the smoothed and projected field $\hat{\tilde{\xi}}$ is used.

The application of the smoothing filter and projection operator requires a modification of the sensitivities given as

$$
\begin{aligned}
\frac{\mathrm{d} \Phi}{\mathrm{d} \xi_{i}} & =\sum_{h \in \mathcal{B}_{e, i}} \frac{\partial \tilde{\xi}_{h}}{\partial \xi_{i}} \frac{\partial \hat{\tilde{\xi}}_{h}}{\partial \tilde{\xi}_{h}} \frac{\mathrm{d} \Phi}{\mathrm{d} \tilde{\tilde{\xi}}_{h}}, \\
\frac{\partial \tilde{\xi}_{h}}{\partial \xi_{i}} & =\frac{w\left(\mathbf{x}_{h}-\mathbf{x}_{i}\right) A_{i}}{\sum_{j \in \mathcal{B}_{e, h}} w\left(\mathbf{x}_{h}-\mathbf{x}_{j}\right) A_{j}}, \\
\frac{\partial \hat{\tilde{\xi}}_{h}}{\partial \tilde{\xi}_{h}} & =\frac{\beta \operatorname{sech}^{2}\left(\beta\left(\tilde{\xi}_{h}-\eta\right)\right)}{\tanh (\beta \eta)+\tanh (\beta(1-\eta))} .
\end{aligned}
$$

Here $\mathcal{B}_{e, i}$ denotes the indices of the design variables, $\xi_{e}$, which are within the filter radius of design variable $\xi_{i}, \mathbf{x}_{(\cdot)}$ denotes the coordinate of the center of element $(\cdot)$ and $A_{(\cdot)}$ is the area of the $(\cdot)$ 'th finite element.

The final stopping criterion used in the optimization procedure is given as

$$
\beta>1000 \wedge\left|\Phi_{n+1}-\Phi_{n}\right|<10^{-3} \cdot \Phi_{n},
$$

where $\Phi_{n}$ is the objective value at the $n$ 'th iteration.

\section{The "Refractive Index" and the Evaluation of $\psi_{\text {target }}$}

Snell's law, (21), relates the refractive index, $n$, of a material placed in vacuum to the incident angle, $\theta_{1}$, and the refracted angle, $\theta_{2}$, of a plane wave impinging on the material as

$n=\frac{\sin \left(\theta_{1}\right)}{\sin \left(\theta_{2}\right)} \Leftrightarrow \theta_{2}=\arcsin \left(\frac{\sin \left(\theta_{1}\right)}{n}\right)$.

Fig. 3 provides an illustration of the negative refraction of a beam, from a slab of material (located in $\Omega_{\mathrm{d}}$ ). The beam enters the domain, $\boldsymbol{\Omega}_{\mathrm{T}}$, at the lower edge centered at $\left\langle x_{0}, y_{0}\right\rangle$. It is refracted with full transmission at the lower interface of the material slab, continues through the slab and is again refracted with full transmission at the top interface. By convention $\theta_{1}$ is measured positivity and $\theta_{2}$ is measured negatively as shown in Fig. 3 .

By using (21) and the sketch in Fig. 3 it is straight forward to derive an expression for the $\mathrm{x}$-coordinate, $x_{3}$, of the center of the beam exiting the MM, based on the following parameters $\left\{x_{0}, y_{0}, y_{1}, y_{2}, y_{3}, \theta_{1}, \theta_{2}\right\}$. I.e the position of the center of

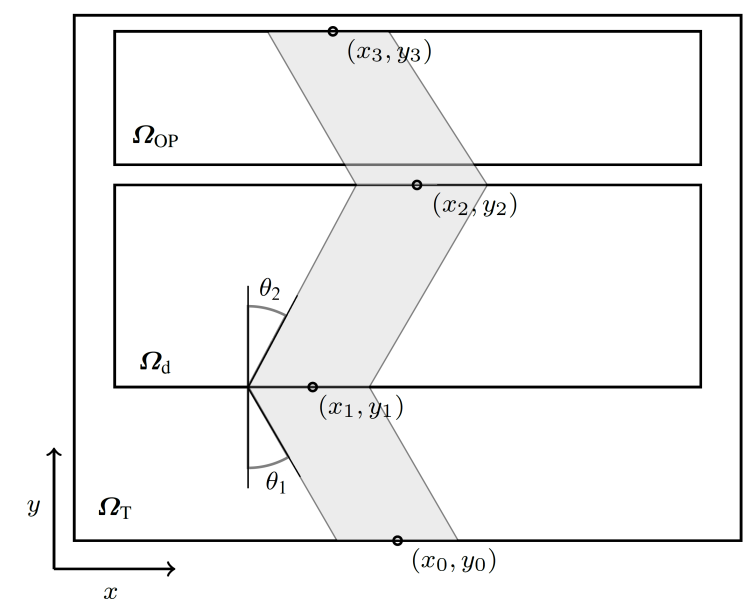

Fig. 3: Sketch of negative refraction of a beam entering the domain centered at $\left\langle x_{0}, y_{0}\right\rangle$, including quantities needed to calculate the placement of the center of $\psi_{\text {target }},\left\langle x_{3}, y_{3}\right\rangle$, in order to obtain a desired $n$ for a given $\theta_{1}$.

the incoming beam $\left\langle x_{0}, y_{0}\right\rangle$, the position of the interfaces of the material slab, $y_{1}, y_{2}$, the angle of incidence, $\theta_{1}$, the angle of refraction, $\theta_{2}$, and the $y$-coordinate of the center of the target wave, $y_{3}$.

$$
\begin{aligned}
x_{3}=x_{0} & +\tan \left(\theta_{1}\right)\left(y_{3}-y_{2}+y_{1}-y_{0}\right) \\
& +\tan \left(\theta_{2}\right)\left(y_{2}-y_{1}\right) .
\end{aligned}
$$

The procedure for evaluating the target wave, $\psi_{\text {target }}$, needed for the optimization problem (10)-(11) for a desired $n$ at a given $\theta_{1}$ and $f$ may now be described as follows.

1. Select the target $n, \theta_{1}$ and $f$.

2. Calculate the refracted angle $\theta_{2}$ using (21).

3. Select the dimensions and position of $\boldsymbol{\Omega}_{\mathrm{T}}, \Omega_{\mathrm{OP}}, \Omega_{\mathrm{d}}$ (this provides $\left.\left\{y_{0}, y_{1}, y_{2}, y_{3}\right\}\right)$.

4. Select the position of the center of the enveloped plane wave $\psi$ generated at $\delta \boldsymbol{\Omega}_{\mathrm{PW}}: \mathbf{x}_{w_{\mathrm{PW}}}=\left\langle x_{0}, y_{0}\right\rangle$.

5. Calculate the $\mathrm{x}$-coordinate of the position of $\psi_{\text {target }}, x_{3}$, using 22,

6. Select the width of $\psi_{\text {target }}: \delta_{x_{\text {target }}}=\delta_{x_{\mathrm{PW}}}$.

7. Evaluate $\psi_{\text {target }}$ using (4)-(5).

Note that the definition of $n$ from Snell's law is not guaranteed to coincide with the definition of the refractive index given as $n=\frac{c}{c_{p}}$, where $c$ is the wave speed in vacuum and $c_{p}$ is the phase velocity of the wave in the medium through which it propagates. That is, it is possible to observe negative refraction of the wave without the resulting field inside the slab having a negative phase velocity. 


\section{Transmittance of Sound Power}

A strength of the proposed objective function is the high level of transmission obtained for the designed meta material slabs. The measure of the transmission through the full MM slab used in the following sections is the transmittance, $T . T$ is obtained by integrating the incoming and transmitted field at the second interface of a slab of MM using the following expression:

$T=\frac{\int_{\delta_{\Omega_{d}}} \psi_{\mathrm{S}}\left(x, y_{2}\right) \overline{\psi_{\mathrm{S}}\left(x, y_{2}\right)} \mathrm{d} x}{\int_{\delta_{\Omega_{d}}} \psi_{\mathrm{E}}\left(x, y_{2}\right) \overline{\psi_{\mathrm{E}}\left(x, y_{2}\right)} \mathrm{d} x}$.

Here $y_{2}$ denotes the $y$-coordinate of the second interface of the slab, see Fig. 3, $\delta_{\Omega_{d}}$ denotes the x-coordinate interval along the second interface of the slab of MM. $\psi_{\mathrm{S}}(y, x)$ denotes the field in the modeling domain containing the MM slab, and $\psi_{\mathrm{E}}(x, y)$ denotes the incident wave in the domain without the slab.

\section{Important Observations}

It is stressed that not all initial configurations lead to final designs with a low objective value, i.e. a MM slab with high $T$ and near the target $n_{\text {target }}$. Tuning the unit cell dimensions relative to the target frequency is necessary to obtain highly performing results. This tuning was performed by numerical experimentation. For the examples considered in the following, a wave length on the order of two times the unit cell size was found to be a good choice. Also, starting the optimization process with a small spatial overlap between $\psi$ and $\psi_{\text {target }}$ was found to be important for the optimization to converge to well-performing final designs. Requiring this overlap sets bounds on the values of $\theta_{1}$ and $n$ for which it is possible to start the optimization. These bounds may be calculated from the width of the Gaussian envelope and the thickness of the MM slab used in the optimization using simply trigonometry. By applying a continuation approach where $\theta_{1}$ and/or $n$ is increased during the optimization it is possible to extend their values beyond those bounds.

It has been found that dividing the optimization process in to two phases, where a restriction is imposed on the design domain in the first phase (Fig. 4i), while the second phase is unrestricted (Fig. 4ii), yields a significant improvement in the convergence of the method to highly performing designs. The restriction used in the numerical examples presented in section 7 consists of restricting the designable part of $\boldsymbol{\Omega}_{\mathrm{d}}$ to an FCC crystal-like grid. The radius of the inclusions in the FCC grid relative to the unit cell size is denoted $r_{\text {FCC }}$. During the first phase all $\xi_{i}$ outside the designable part are fixed at $\xi_{i}=0$, while in the second phase all $\xi_{i}$ are allowed to change. Fig. 4 illustrates the designable part of $\Omega_{\mathrm{d}}$, highlighted in gray, in the two phases using a sample $4 \times 3$ unit cell slab.

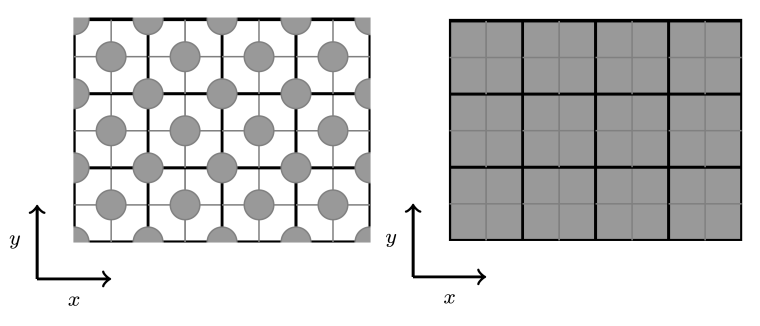

(i)

(ii)

Fig. 4: Sketch of a (i) Restricted and (ii) Free periodic design domain consisting of $4 \times 3$ doubly axis symmetric unit cells.

For all examples presented in the paper $r_{\mathrm{FCC}}=0.25$ is used and the duration of the two phases is linked to the $\beta$-continuation scheme. Phase one is executed for $\beta \leq 4$ and phase two is executed for $\beta>4$.

By tuning the unit cell size and using the two phase approach the proposed method has been found to converge to MMs with low objective value, i.e. $T>0.95$ and $n$ within $\approx 10 \%$ of $n_{\text {target }}$ when evaluated at the target $\theta_{1}$ and $n$, for all tested values of $n \in[-4,-0.5]$ and $\theta_{1} \in\left[2.5^{\circ}, 15^{\circ}\right]^{1}$. Designing MMs for values of $n$ and/or $\theta_{1}$ outside the range stated above may be done by applying a continuation approach for $\theta_{1}$ and the position of $\psi_{\text {target }}, \mathbf{x}_{w_{\text {target }}}$. In the continuation scheme $\theta_{1}$ and/or $n$ are changed gradually from an initial value to the final desired value during the optimization. Applying the continuation approach with four changes of $\theta_{1}$ and/or $n$ during the initial part of the optimization with 20 iteration between each change, it has been found possible to design MMs with $T>0.85$ and within $\approx 25 \%$ of the desired $n$ for $n \in[-4,-0.35]$ for $\theta_{1}=10^{\circ}$ and for $\theta_{1} \in\left[2.5^{\circ}, 30^{\circ}\right]$ for $n=-1$. The reason for the upper limits on $\theta_{1}$ and $n$ when using the continuation scheme is that the width of the slab becomes a limiting factor (a wider slab is more expensive from a computational perspective). The authors find it reasonable to expect that MMs may be designed for larger $\theta_{1}$ and/or $n$ closer to zero if the width of the slab is increased.

\section{Numerical Results}

Numerical results demonstrating the proposed method are presented. The material parameters for the solid

${ }^{1}$ Note that the proposed formulation does not allow $\theta_{1}=0^{\circ}$ due to the formulation relying on 21 . 
and void regions are chosen to be those of aluminum; $\rho_{\text {solid }}=2630.0 \mathrm{~kg} \mathrm{~m}^{-3}, \kappa_{\text {solid }}=6.87 \cdot 10^{10} \mathrm{~N} \mathrm{~m}^{-2}$ and air; $\rho_{\text {air }}=1.204 \mathrm{~kg} \mathrm{~m}^{-3}, \kappa_{\text {air }}=141.921 \cdot 10^{3} \mathrm{~N} \mathrm{~m}^{-2}$, respectively. This provides a high contrast between solid and void resulting in near perfect reflection of the acoustic waves from solid regions, corresponding to a zero Neumann boundary condition along solid/void interfaces. The high impedance mismatch between the air and solid ensures that no significant excitation of elastic waves occurs in the solid material. Thus in the present case, where aluminum and air are considered, the elastic-acoustic interactions may be ignored, as is the standard procedure when performing topology optimization of high contrast acoustic problems (Dühring et al, 2008). The authors have investigated and validated this assumption both numerically and experimentally in previous work, concerning a topology optimized acoustic cavity (Christiansen et al, 2015a).

The parameter values used in the numerical examples are given in table 1 . The designable MM slab consists of $22 \times 6$ unit cells. Each unit cell is discretized into $80 \times 80$ finite elements, with each element containing a single design variable, yielding a total of 844800 finite elements in $\Omega_{\mathrm{d}}$ and 3600 design variables. If the full domain $\Omega$ is discretized using a FEM method $\approx 3 \cdot 10^{6}$ finite elements are needed yielding $\approx 3 \cdot 10^{6}$ degrees of freedom. Using the Hybrid WBM-FEM method the total number of degrees of freedom is reduced to $\approx 0.88 \cdot 10^{6}$, at the expense of a small fraction of the system matrix being dense. The software used to solve the problem is written in MATLAB. When executing the code on a single $2.40 \mathrm{GHz}$ Intel Core i7-3630 QM processor for the chosen parameters optimizing for one $\theta_{1}$ and one $f$ a design iteration uses 100-300 seconds (depending on the number of inner iterations in GCMMA). A total of 200-250 design iterations are used for the investigated cases.

For all examples an enveloped plane wave with the nondimensionalized wavelength, $\lambda=\frac{1}{3}$, is excited at $\delta \boldsymbol{\Omega}_{\mathrm{PW}}$. The unit cell size is $\left(a_{x}, a_{y}\right)=\left(\frac{1}{6}, \frac{1}{6}\right)$ in non-dimensional units; hence there are 2 unit cells per free space wavelength of the pressure field. The performance of the designed MMs in terms of $n$ and $T$ is in all cases investigated by considering a slab consisting of $22 \times 12$ unit cells, i.e. a slab of twice the depth of the slab used in the optimization procedure. The increased depth is used to highlight that the optimized design works as a bulk material and not only for the thickness of the slab used in the optimization. That is, the performance of the designed MM slabs consisting of the optimized unit cell designs show little dependence on slab thickness.

\begin{tabular}{ll}
\hline Parameter & Value \\
\hline$x_{\boldsymbol{\Omega}_{\mathrm{T}}} \times y_{\boldsymbol{\Omega}_{\mathrm{T}}}$ & {$[-26 / 12,26 / 12] \times[-18 / 12,18 / 12]$} \\
$x_{\boldsymbol{\Omega}_{\mathrm{op}}} \times y_{\boldsymbol{\Omega}_{\mathrm{op}}}$ & {$[-26 / 12,26 / 12] \times[8 / 12,18 / 12]$} \\
$x_{\boldsymbol{\Omega}_{\mathrm{d}}} \times y_{\boldsymbol{\Omega}_{\mathrm{d}}}$ & {$[-22 / 12,22 / 12] \times[-6 / 12,6 / 12]$} \\
$\mathcal{N}_{\mathrm{e}, \mathrm{x}} \times \mathcal{N}_{\mathrm{e}, \mathrm{y}}$ & $80 \times 80$ \\
$\mathcal{N}_{\mathrm{uc}, \mathrm{x}} \times \mathcal{N}_{\mathrm{uc}, \mathrm{y}}$ & $22 \times 6$ \\
$\mathbf{x}_{w_{\mathrm{PW}}}$ & $\langle 0.0,-26 / 12\rangle$ \\
$\delta_{\mathrm{x}, \mathrm{PW}}=\delta_{\mathrm{x}, \mathrm{target}}$ & 0.7 \\
$\delta \boldsymbol{\Omega}$ & $\delta \boldsymbol{\Omega}_{\mathrm{T}}$ \\
$\delta \boldsymbol{\Omega}_{\mathrm{PW}}$ & {$[-26 / 12,26 / 12] \times-18 / 12$} \\
$R$ & $\frac{1}{240}$ \\
$\eta$ & 0.5 \\
$V$ & 0.25 \\
$c_{s}$ & $10^{3}$ \\
\hline
\end{tabular}

Table 1: $x_{[\cdot]} \times y_{[\cdot]}$ : Non-dimensional domain sizes. $\mathcal{N}_{\mathrm{e}, \mathrm{x}} \times$ $\mathcal{N}_{\mathrm{e}, \mathrm{y}}$ : Number of finite elements per unit cell. $\mathcal{N}_{\mathrm{uc}, \mathrm{x}} \times \mathcal{N}_{\mathrm{uc}, \mathrm{y}}$ : Number of unit cells in the material slab. $\mathbf{x}_{w_{\mathrm{PW}}}$ : Center of incoming wave. $\delta_{\mathrm{x}, \mathrm{PW}}$ : Width of Gaussian envelope on incoming wave. $\delta_{\mathrm{x}, \text { target }}$ : Width of Gaussian envelope on target wave. $\delta \boldsymbol{\Omega}_{[\cdot]}$ : Domain boundary. $R$ : Filter radius. $\eta$ : Projection level. $V$ : Volume constraint. $c_{s}$ : Objective function scaling.

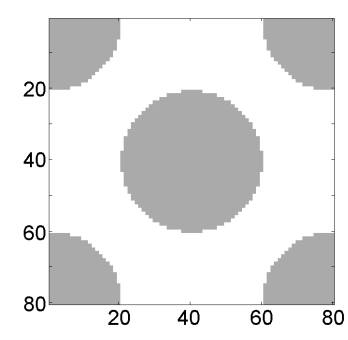

(i)

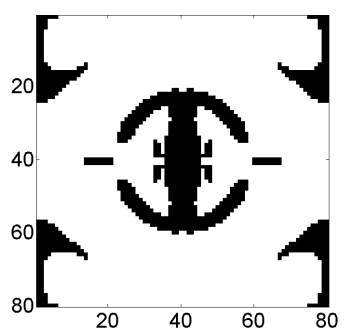

(ii)
Fig. 5: (i) Initial guess for a unit cell in the restricted design. (ii) Unit cell in the final design for the example in section 7.1

\subsection{Single Angle of Incidence, $n_{\text {target }}=-1$}

As a first demonstration of the proposed method, we consider designing a MM for the target negative refractive index, $n_{\text {target }}=-1$, at the incident angle, $\theta_{1}=10^{\circ}$ and the non-dimensional frequency $f=3$.

Fig. 5i shows the initial guess for a unit cell in the design domain, where the gray area corresponds to $\xi=0.25$ and the white area to $\xi=0$. Fig. 5ii shows a unit cell in the final design where black is material $(\hat{\tilde{\xi}}=1)$ and white is void 


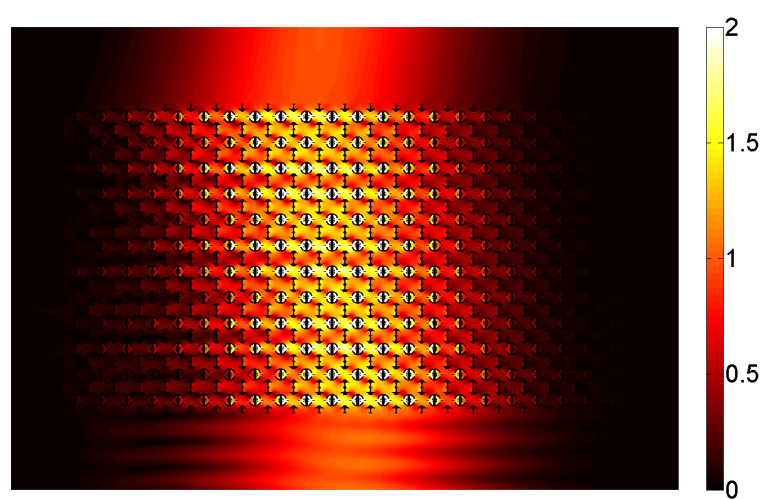

(i)

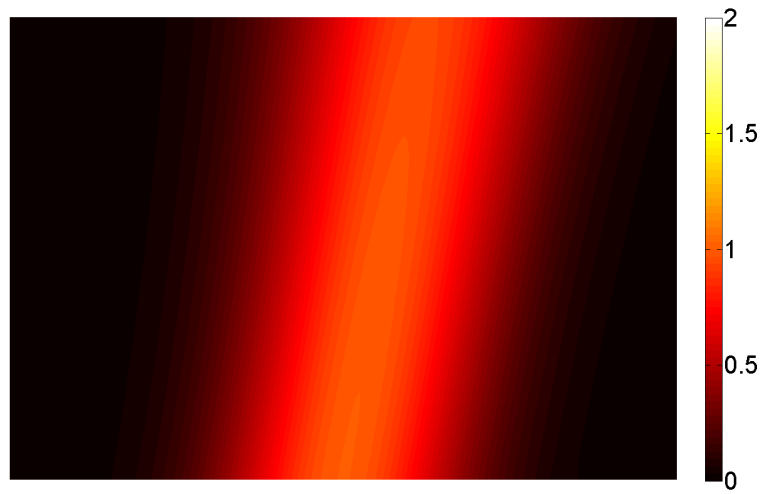

(ii)

Fig. 6: (color online) Amplitude of the solution to the model problem (1)-(3), $|\psi|$, in $\boldsymbol{\Omega}_{\mathrm{T}}$ for an incident angle $\theta_{1}=10^{\circ}$ (i) containing a MM slab of $22 \times 12$ unit cells consisting of the optimized design in Fig. 5ii (ii) In an empty $\boldsymbol{\Omega}_{\mathrm{T}}$.

$(\hat{\tilde{\xi}}=0)$.

Fig. 6 shows the amplitude of the pressure field, $|\psi|$, in $\boldsymbol{\Omega}_{\mathrm{T}}$ for an incident enveloped plane wave at $\theta_{1}=10^{\circ}$, Fig. 6i with and Fig. 6ii without the MM slab placed in the center of the modeling domain.

The refractive index of the MM slab is estimated by applying the following procedure. The field inside the MM, $\psi$, is fitted to a perfect Gaussian envelope, $\psi_{\mathrm{PGE}}$ :

$\begin{aligned} \psi_{\mathrm{PGE}} & =A_{\mathrm{PGE}} \mathrm{e}^{-\left(\frac{\left(\mathbf{x}-\mathbf{x}_{w}\right) \cdot \hat{\mathbf{d}}}{\delta_{x}}\right)^{2}} \\ & =A_{\mathrm{PGE}} \mathrm{e}^{-\left(\frac{-\left(x-x_{w}\right) \sin \left(\pi / 2+\theta_{2, \mathrm{fit}}\right)+\left(y-y_{w}\right) \cos \left(\pi / 2+\theta_{2, \mathrm{fit}}\right)}{\delta_{x}}\right)^{2}},\end{aligned}$

whose width $\delta_{x}$, amplitude $A_{\mathrm{PGE}}$, position $\mathbf{x}_{w}$, and angle for the direction of propagation $\theta_{2 \text {,fit }}$ are free parameters. The fit is made using a nonlinear least-squares curve fitting tool which solves the minimization problem; $\min _{\delta_{x}}\left\|\psi-\psi_{\mathrm{PGE}}\right\|_{2}^{2}$ over the meta material slab.
The angle of refraction of the incoming enveloped plane wave, $\theta_{2} \approx \theta_{2, \text { fit }}$, is obtained from the fit.

The angle $\theta_{2, \text { fit }}$ obtained from the fit along with the angle $\theta_{1}$ of the incoming wave is used to estimate $n$ using (21). It is noted that $\theta_{2, \text { fit }}$ in some cases were found to be sensitive to the initial guess of the fitting parameters in the fit with a maximal variation of $\Delta \theta_{2}=0.5^{\circ}$. Hence a bound on $\theta_{2} \approx \theta_{2, \text { fit }}$ is $\theta_{2, \text { bound }} \in\left[\theta_{2, \text { fit }}-\Delta \theta_{2}, \theta_{2, \text { fit }}+\Delta \theta_{2}\right]$. The uncertainty in $\theta_{2}$ propagates to uncertainty in the refractive index. Therefore bounds on $n$ have been calculated, providing an estimate of a minimum value, $n_{\min }=\frac{\sin \left(\theta_{1}\right)}{\sin \left(\theta_{2, \text { fit }}+\Delta \theta_{2}\right)}$ and maximum value $n_{\max }=\frac{\sin \left(\theta_{1}\right)}{\sin \left(\theta_{2, \text { fit }}-\Delta \theta_{2}\right)}$ for $n$ for a given $\theta_{1}$. These bounds are included in all reported values of $n$.

Using this procedure for the present case one obtains $n \approx-1.06 \pm 0.05$ and $T \approx 0.98$ for the bulk $\mathrm{MM}$ at the prescribed frequency $f=3$ and angle of incidence $\theta_{1}=10^{\circ}$. Hence the designed acoustic MM is exhibiting the desired negative refraction to within $\approx 10 \%$ and with high transmission at the prescribed angle of incidence.

\subsubsection{Angular and Frequency Dependence}

An investigation of the angular and frequency dependence of $T$ and $n$ are presented in Fig. 7i and 7ii respectively.

Fig. $7 \mathrm{i}$ shows $T\left(\theta_{1}\right)$ for five equidistant frequencies in the interval, $f \in[2.7,3.3]$. Here it is seen that $T$ remains above 0.95 for the frequencies $f \in[2.7,3.0]$ for $\theta_{1} \in\left[2.5^{\circ}, 15^{\circ}\right]$ after which it drops off for all frequencies. For the two higher frequencies the transmission quickly drops off. This is in part due to some of the energy being reflected from the interfaces of the MM and in part due to $n$ going towards zero, i.e. a large value of $\left|\theta_{2}\right|$ which results in part of the energy being refracted towards the left side of the MM slab.

Consider now the refractive index shown in Fig. 7ii for $f \in\{2.85,3.00,3.15,3.30\}$. Here it is seen that $n$ is sensitive towards both angular changes and frequency changes, however it remains negative across all investigated frequencies and angles. $n$ for $f=2.75$ is $-475<n<-17 \forall \theta_{1}$ meaning that the refracted angle, $\theta_{2}$ is in the interval $0.0^{\circ} \leq \theta_{2} \leq 0.5^{\circ}$. Hence for this frequency the refracted field is transmitted through the slab almost without any transverse change in position.

Fig. 8 illustrates the variation in the field across a range of incoming angles, $\theta_{1} \in\left[2.5^{\circ}, 15^{\circ}\right]$ for $f=3.0$. 


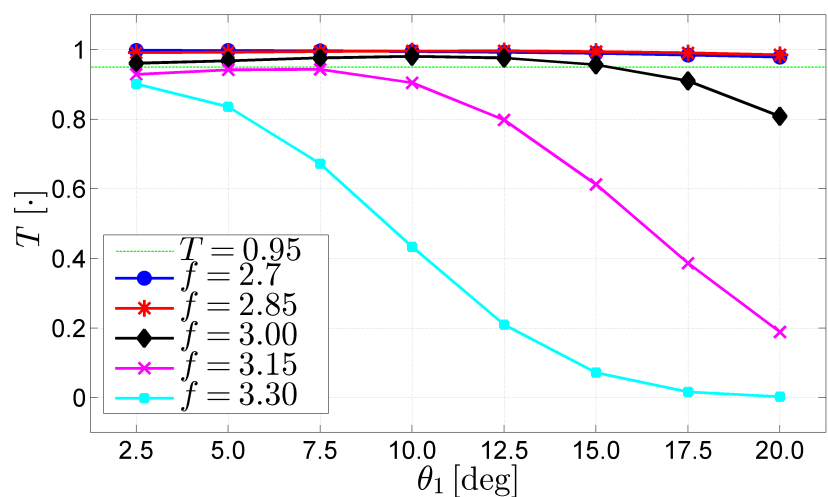

(i)

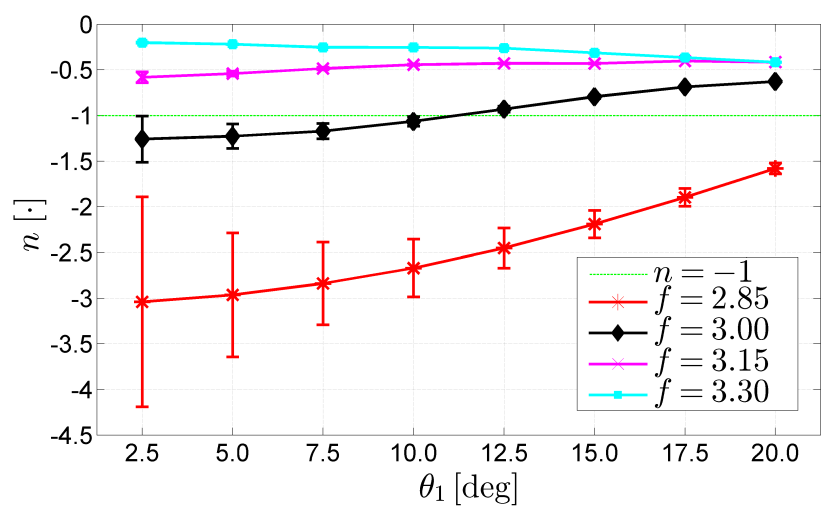

(ii)

Fig. 7: (color online) (i) Transmittance and (ii) refractive index, including error bars denoting the uncertainty in $n$ stemming from the uncertainty in $\theta_{2}$,fit, for MM slab consisting of $22 \times 12$ unit cells containing the optimized design shown in Fig. 5ii as a function of incident angle, $\theta_{1}$, for different frequencies.

\subsubsection{The Field Inside the MM}

By looking at the temporal and spatial variations of the pressure field inside the MM it is observed that the field pattern is non-trivial. I.e. the field inside the MM slab cannot be described exactly by a simple plane wave traveling through the slab. As an example of investigating the field inside the MM slab, consider a plane wave with $f=$ 3 impinging on the meta material slab at a right angle $\left(\theta_{1}=0\right)$. For this investigation infinite periodicity in the $x$-direction is assumed. By performing a Fourier transform, $F(\psi, k)$, of the field inside the MM, utilizing the periodicity to only consider a $1 \times 12$ column of unit cells, and recording the magnitude $|F|^{2}$ for wave vectors, $\mathbf{k}=\left\langle k_{x}, k_{y}\right\rangle$, with zero $x$-component, one obtains the spectrum shown in Fig. 9 From here it is seen that four modes are excited inside the MM. A dominant mode centered at the positive wave number $\operatorname{sgn}\left(k_{y}\right) \cdot\left|k_{p}\right| \approx 3.9 \cdot 2 \pi$ along with a number of side lobes as well as three modes being approximately an order of magnitude smaller with negative wave numbers centered at, $\operatorname{sgn}\left(k_{y}\right) \cdot\left|k_{n_{1}}\right| \approx-2 \cdot 2 \pi, \operatorname{sgn}\left(k_{y}\right) \cdot\left|k_{n_{2}}\right| \approx$

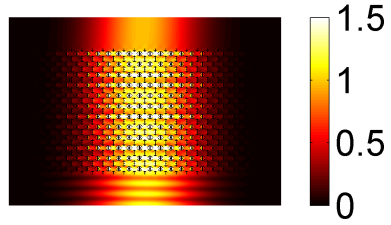

(i)

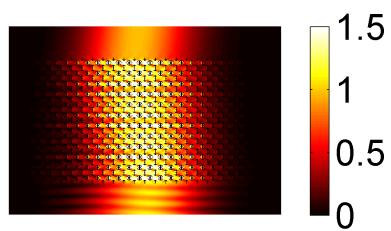

(iii)

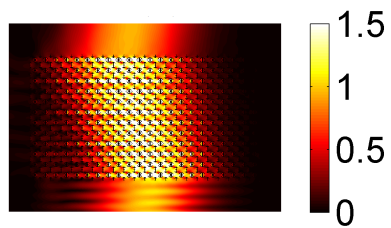

(v)

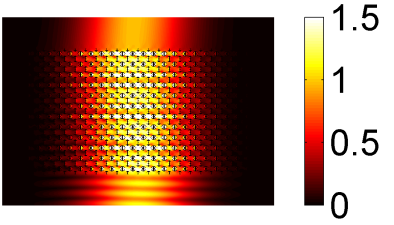

(ii)

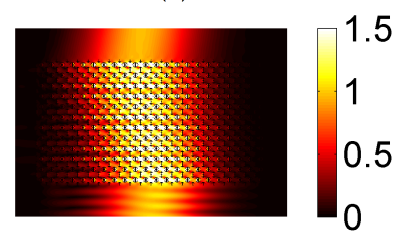

(iv)

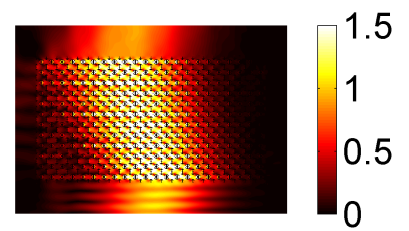

(vi)
Fig. 8: (color online) Amplitude of the solution to the model problem (1)-(3), $|\psi|$, in $\Omega_{\mathrm{T}}$ containing a MM slab of $22 \times 12$ unit cells consisting of the optimized design in Fig. 5iil for six different incident angles $\theta_{1}$ at the target frequency $f=3.0$. (i) $\theta_{1}=2.5^{\circ}$ (ii) $\theta_{1}=5.0^{\circ}$ (iii) $\theta_{1}=7.5^{\circ}$ (iv) $\theta_{1}=10.0^{\circ}$ (v) $\theta_{1}=12.5^{\circ}$ (vi) $\theta_{1}=15.0^{\circ}$.

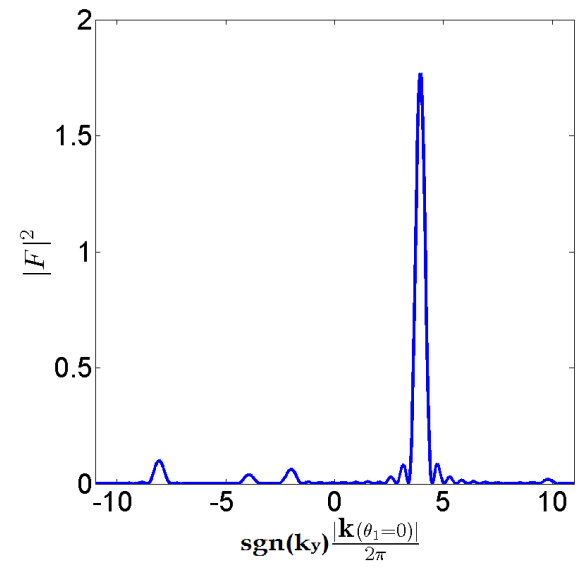

Fig. 9: (color online) Squared magnitude of the Fourier transform of the field inside the MM slab across $1 \times 12$ unit cells for an incident plane wave, $\psi$, at $\theta_{1}=0^{\circ}$ and $f=3$ for the design shown in Fig. 5ii for a k-vector with zero $\mathrm{x}$-component.

$-3.9 \cdot 2 \pi, \operatorname{sgn}\left(k_{y}\right) \cdot\left|k_{n_{3}}\right| \approx-8.1 \cdot 2 \pi$, respectively. This example indicates that the designed MM slab exhibits negative refraction through an interaction of excited waves having both positive and negative phase velocity, $c_{p}=\frac{\omega}{k}$. The finding that several modes contribute to the field inside the MM agrees with the findings in Mortensen et al (2010), where an optical material is considered. 


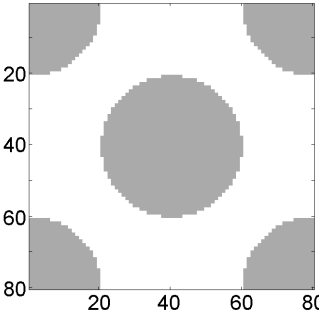

(i)

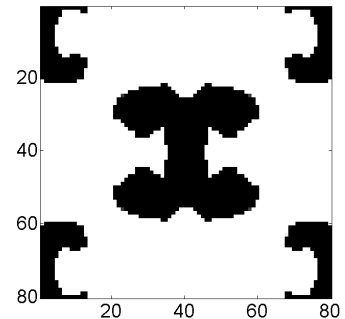

(ii)
Fig. 10: (i) Initial guess for a unit cell in the restricted design. (ii) Unit cell in the final design for the example in section 7.2 Black is solid, white is void and gray is an intermediate between solid and void.

\subsection{Multiple Incoming Angles, $n_{\text {target }}=-1$}

The first example illustrated that the proposed method can be used to design MMs exhibiting a given negative $n$ for a single incoming angle and frequency with good accuracy and high $T$. Furthermore, it was seen that the refractive index remains negative across a wide range of incoming angles and a frequency band of $20 \%$. However, both the refractive index and transmittance exhibit a strong dependence on both $\theta_{1}$ and $f$. This example illustrates that the variation with $\theta_{1}$ may be reduced and the overall performance increased by optimizing the MM across a range of incoming angles. The same parameters as the first example are used in the optimization problem, except that three incident angles are considered simultaneously, $\theta_{1} \in$ $\left\{5^{\circ}, 10^{\circ}, 15^{\circ}\right\}$. Fig. 10ii shows the unit cell for the final design.

Fig. 11i and Fig. 11ii show a comparison of $T$ and $n$, respectively, for the MM seen in Fig. 5ii, denoted $E_{1}$, and the MM seen in Fig. 10ii, denoted $E_{2}$, evaluated at the target frequency $f=3.0$.

From Fig. 11i it is observed that the transmission has improved for all investigated angles such that $T\left(\theta_{1}\right)>$ $0.96 \forall \theta_{1} \in[2.5,15]$ and from Fig. 11 ii it is seen that the variation in $n$ has decreased slightly across $\theta_{1} \in[5,15]$. An increase in transmittance of $\approx .005$ is seen even for the angle $\theta_{1}=10^{\circ}$ for which $E_{1}$ was optimized. The explanation for this unexpected increase is the decrease in $n$ compared to the target value. I.e. in terms of the objective function value for $\left(f, \theta_{1}\right)=\left(3,10^{\circ}\right), E_{1}$ still outperforms $E_{2} ; \Phi_{E_{1}}(f=$ $\left.3, \theta_{1}=10\right) \approx 0.17$ and $\Phi_{E_{2}}\left(f=3, \theta_{1}=10\right) \approx 1.7$.

\subsubsection{Uniform Spatial Variations}

The ultimate goal of designing MM slabs exhibiting negative refraction is using the resulting designs in real world applications. Hence the optimized designs have to be manufactured and regardless of the chosen method,

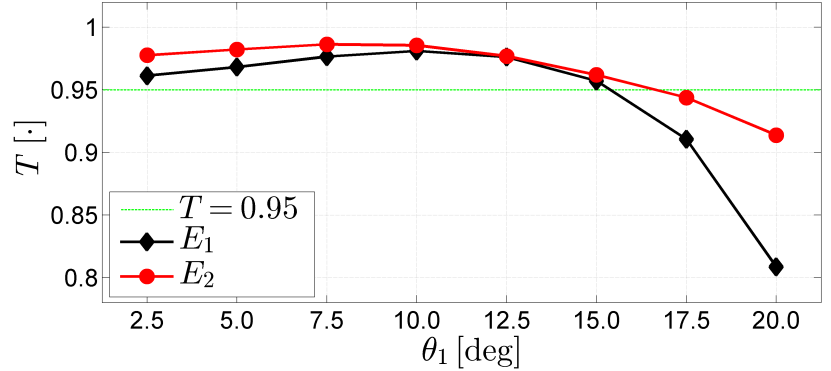

(i)

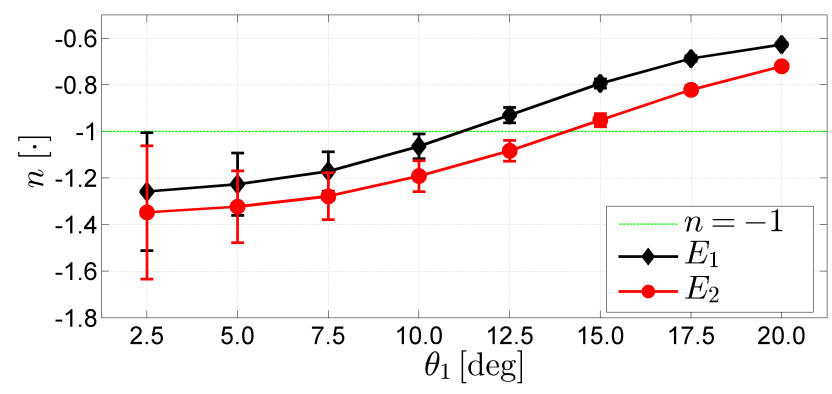

(ii)

Fig. 11: (color online) Comparison of (i) Transmittance and (ii) refractive index, including error bars denoting the uncertainty in $n$ stemming from the uncertainty in $\theta_{2, \text { fit }}$, for MM slab consisting of $22 \times 12$ unit cells seen in Fig. 5 ii $\left(E_{1}\right)$ and a MM slab of $22 \times 12$ unit cells seen in Fig. 10ii $\left(E_{2}\right)$. The frequency used for the calculations is the target frequency for the optimization $f=3$.

production errors may occur. It is therefore valuable to know how production errors affect the designs performance. Common manufacturing errors in micro-nano-processing are over- and under-etching or over- and under exposure which can be observed as near uniform erosion or dilation of manufactured devices (Zhou et al, 2016, Jansen et al 2013). Hence, this section presents an investigation of how uniform dilation/erosion of the optimized design considered in section 7.2, optimized for multiple incoming angles, affects its transmittance and index of refraction. The dilation/erosion of the design is performed by adding/removing a single element of material to/from the edge of each feature, corresponding to $1 / 80$ or $1.25 \%$ of unit cell size. The unit cells for the dilated, $E_{2, \text { Dilate }}$, and eroded, $E_{2, \text { Erode }}$, design are presented in Fig. 12 along with illustrations of the changes from the optimized unit cell seen in Fig. 10ii For the eroded design the narrow features along the top and bottom edges of the unit cell disappear completely, while for the dilated design all features remain intact.

Fig. 13 shows the amplitude of the pressure field $|\psi|$ in $\Omega_{\mathrm{T}}$ for an enveloped plane wave incident at $\theta_{1}=10^{\circ}$ for the eroded, optimized and dilated design. Changes in both $n$ and $T$ introduced by the erosion/dilation can be observed.

An investigation of $n\left(\theta_{1}\right)$ and $T\left(\theta_{1}\right), \theta_{1} \in\left[0^{\circ}, 20^{\circ}\right]$, for $E_{2}, E_{2, \text { Erode }}$ and $E_{2, \text { Dilate }}$ is presented in Fig. 14. Both 


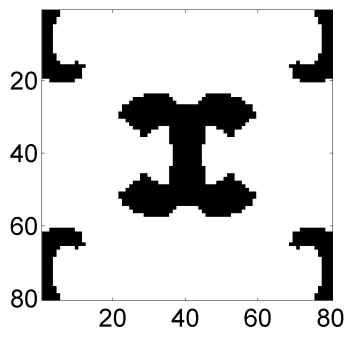

(i)

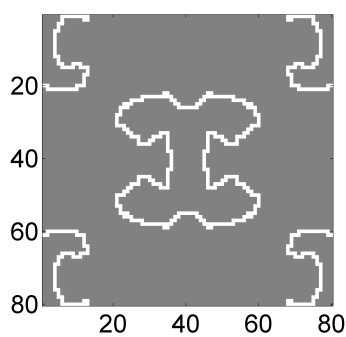

(iii)

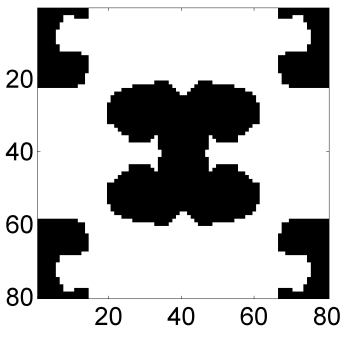

(ii)

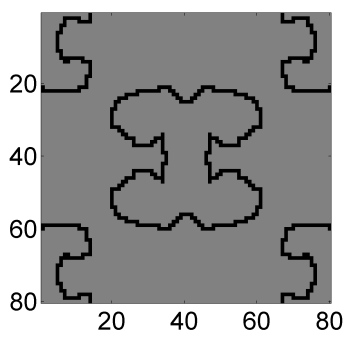

(iv)
Fig. 12: (i) Eroded, $E_{2, E r o d e}$, and (ii) Dilated, $E_{2, \text { Dilate }}$, unit cell for the optimized design, $E_{2}$, presented in Fig. 10ii Here black denotes material and white denotes void. (iii) and (iv) show the difference between the optimized design and the eroded and dilated designs respectively. Here gray denotes unchanged areas of the unit cell while white denotes removed material and black denotes added material.

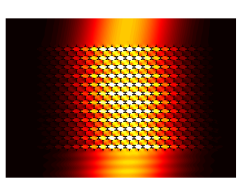

(i)

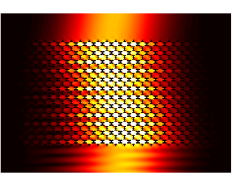

(ii)

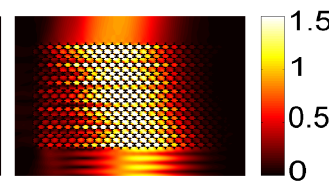

(iii)
Fig. 13: (color online) Amplitude of the solution to the model problem (1)-(3), $|\psi|$, with $\theta_{1}=10^{\circ}$, in $\Omega_{\mathrm{T}}$ containing a MM slab of $20 \times 12$ unit cells consisting of the (i) eroded, (ii) optimized and (iii) dilated design presented in Fig. 12i 10ii and 12ii respectively.

$n\left(\theta_{1}\right)$ and $T\left(\theta_{1}\right)$ are affected by the dilation/erosion, which is not unexpected as the design was not optimized for spatial robustness. Although the performance of the MM slab deteriorates in both cases the index of refraction remains negative for all $\theta_{1}$ and $T>0.8$ for $\theta_{1}<15^{\circ}$. Considering $E_{2, \text { Erode }}, T$ is observed to increase for all $\theta_{1}$, whereas $n$ deteriorates compared to $E_{2}$ and the optimization target $n_{\text {target }}=-1$. For $E_{2, \text { Dilate }}$ the transmittance decreases and the index of refraction increases, i.e. $\theta_{2}$ increases for all $\theta_{1}$. It is interesting to note that the erosion and the dilation have opposite effects on $T$ and $n$, with $T$ increasing and $n$ decreasing for the erosion and vise versa for the dilation.

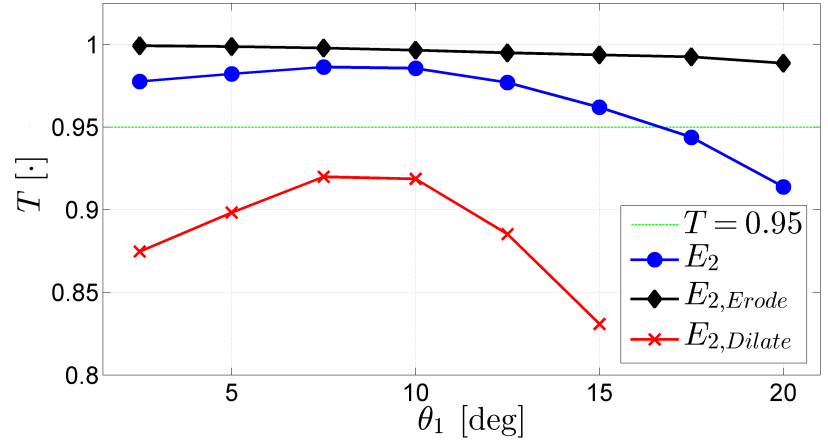

(i)

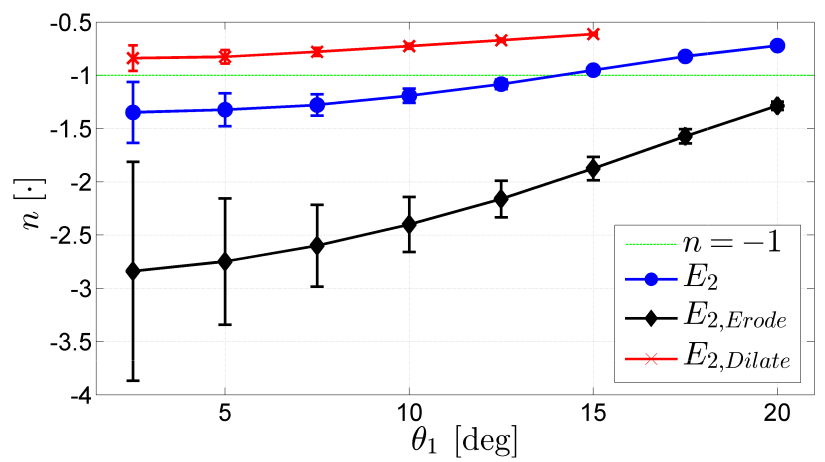

(ii)

Fig. 14: (color online) (i) Transmittance and (ii) refractive index, including error bars denoting the uncertainty in $n$ stemming from the uncertainty in $\theta_{2}$,fit, as a function of $\theta_{1}$ for a MM slab consisting of $22 \times 12$ unit cells of the optimized $E_{2}$, eroded $E_{2, \text { Erode }}$ and dilated $E_{2, \text { Dilate }}$ designs presented in Fig. 10ii Fig. 12i and Fig. 12ii respectively.

\subsection{Frequency Band, $n_{\text {target }}=-1$}

The example in section 7.2 demonstrated that it is possible to increase the transmission and reduce the variation in $n$ across an interval of incoming angles by accounting for multiple angles in the design process. The next example investigates if it is possible to obtain similar results across a frequency band as well. Consider the same parameters used in the first example except that the design is optimized for three wave lengths simultaneously. The nondimensional wavelengths are $\lambda_{i} \in\{10 / 27,10 / 30,10 / 33\}$ corresponding to $f \in\{2.85,3,3.15\}$ i.e. a $10 \%$ frequency band centered at $f_{c}=3$. The resulting unit cell design is shown in Fig. 15ii.

Fig. 16 shows a comparison of $T$ and $n$ between the MM shown in Fig. 5ii optimized for a single frequency, denoted $E_{1}$, and the MM shown in Fig. 15ii optimized for the three frequencies $f \in[2.85,3.00,3.15]$, denoted $E_{3}$, both at the incoming angle $\theta_{1}=10^{\circ}$.

Considering the transmittance in Fig. 16i it is seen that it oscillates for both $E_{1}$ and $E_{3}$. For $E_{3}$ the oscillations have peaks at the three frequencies for which the MM slab was 


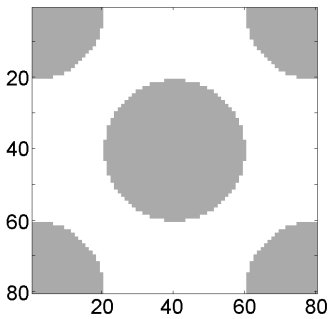

(i)

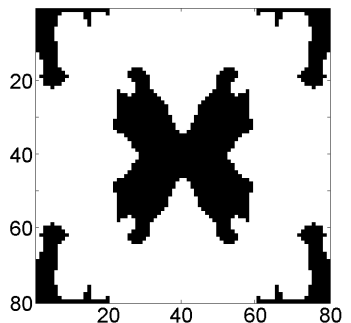

(ii)
Fig. 15: (i) Initial guess for a unit cell in the restricted design. (ii) Unit cell in the final design for the example in section 7.3 Black is solid, white is void and gray is an intermediate between solid and void.

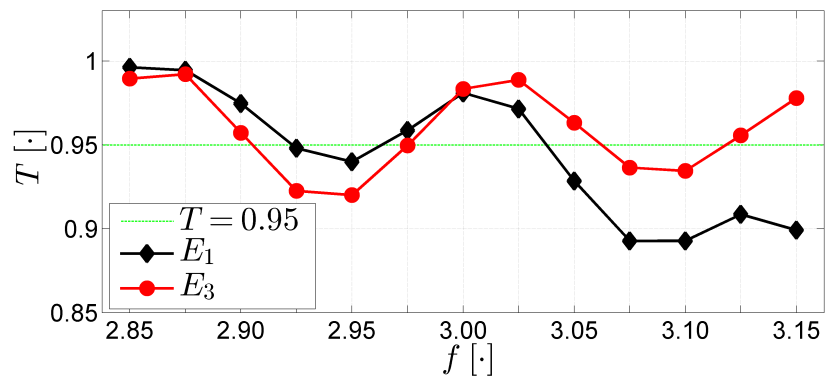

(i)

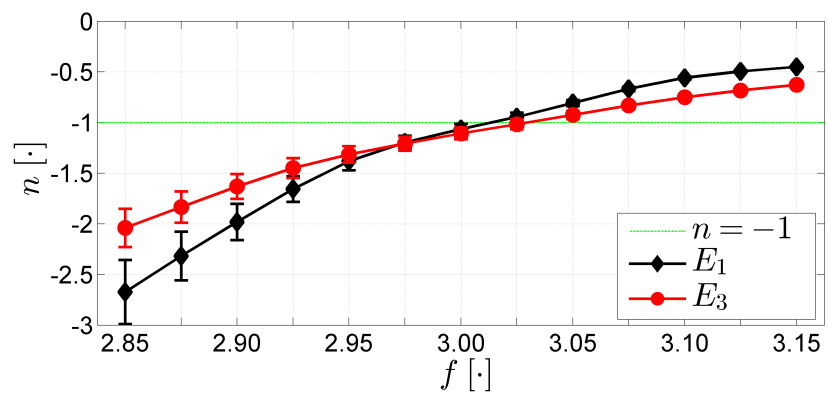

(ii)

Fig. 16: (color online) Comparison of (i) Transmittance and (ii) refractive index, including error bars denoting the uncertainty in $n$ stemming from the uncertainty in $\theta_{2, \text { fit }}$, for MM slab consisting of $22 \times 12$ unit cells seen in Fig. 5ii ( $\left.E_{1}\right)$ and a MM slab of $22 \times 12$ unit cells seen in Fig. 15ii $\left(E_{3}\right)$ as a function of frequency, $f$, for the incident angle $\theta_{1}=10^{\circ}$.

designed (as is to be expected). Calculating the average transmittance from Fig. 16i across the frequency interval $f \in[2.85,3.15]$ one gets $\left\langle T_{E_{3}}\right\rangle \approx 0.960$ and $\left\langle T_{E_{1}}\right\rangle \approx 0.945$ for the frequency band and single frequency optimization respectively. An improvement of $\approx 0.015$. If one considers the worst case transmittance it has seen to improve from $\min \left(T_{E_{1}}\right) \approx 0.893$ to $\min \left(T_{E_{3}}\right) \approx 0.920$. Regarding the refractive index Fig. 16iil shows a reduction in the variation of $n$ with frequency as well as an overall improvement in accuracy compared to the target $n_{\text {target }}=-1$. Although the accuracy at $f=3.0$ has decreased by $\approx 4 \%$, it has increased

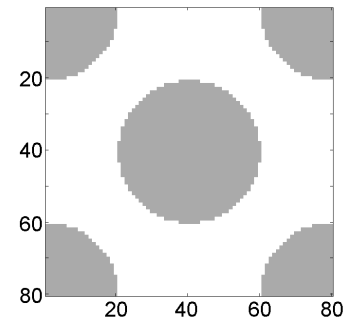

(i)

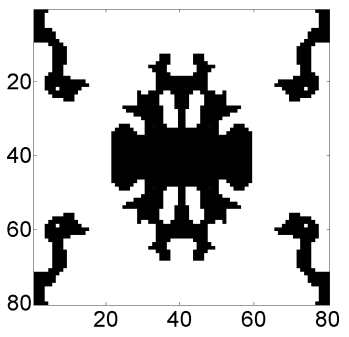

(ii)
Fig. 17: (i) Initial guess for a unit cell in the restricted design. (ii) Unit cell in the final design for the example in section 7.4 Black is solid, white is void and gray is an intermediate between solid and void.

in the rest of the interval. At $f=3.15$ the increase is $\approx 40 \%$ while at $f=2.85$ the increase is between $6 \%$ and $38 \%$ when the uncertainty in $n$, caused by the uncertainty in $\theta_{2, \text { fit }}$, is taken into account.

7.4 Multiple Incoming Angles, $n_{\text {target }}=-0.5$

The final example shows the design for an MM optimized for $n_{\text {target }}=-0.5$ and the angular range, $\theta_{1} \in\left[2.5^{\circ}, 7.5^{\circ}\right]$. Three target angles $\theta_{1} \in\left\{2.5^{\circ}, 5.0^{\circ}, 7.5^{\circ}\right\}$ are used in the optimization. All other parameters are the same as those used in the first example. The resulting design is presented in Fig. 17ii The dependence of $T$ and $n$ on incoming angle and frequency is illustrated in Fig. 18. An illustration of the field in and around the MM slab as a function of $\theta_{1}$ is presented in Fig. 19 By comparing the field in Fig. 8 and Fig. 19 the change in $n$ for the two MM slabs is observed.

Fig. 18i shows $T\left(\theta_{1}\right)$ for three equidistant frequencies in the interval, $f \in[2.85,3.15]$. $T$ remains above 0.95 for the frequencies $f \in[2.85,3.0]$ for $\theta_{1} \in\left[1.5^{\circ}, 7.5^{\circ}\right]$. For $f=3.15$ the transmission quickly drops off. As for the first examples this is in part due to some of the energy being reflected from the interfaces of the MM and in part due to $n_{f=3.15} \approx-0.25$ which results in $\left|\theta_{2}\right|$ growing quickly, in turn resulting in part of the energy being refracted towards the left side of the MM slab. Considering the refractive index shown in Fig. 18ii for $f \in\{2.85,3.00,3.15\}$, it is seen that $n$ varies with both $f$ and $\theta_{1}$ while remaining negative across all investigated values. The variations in $n$ are larger with $f$ than with $\theta_{1}$. Compared to the first example the variations in both $f$ and $\theta_{1}$ are slightly smaller across the investigated range of $\theta_{1}$. 


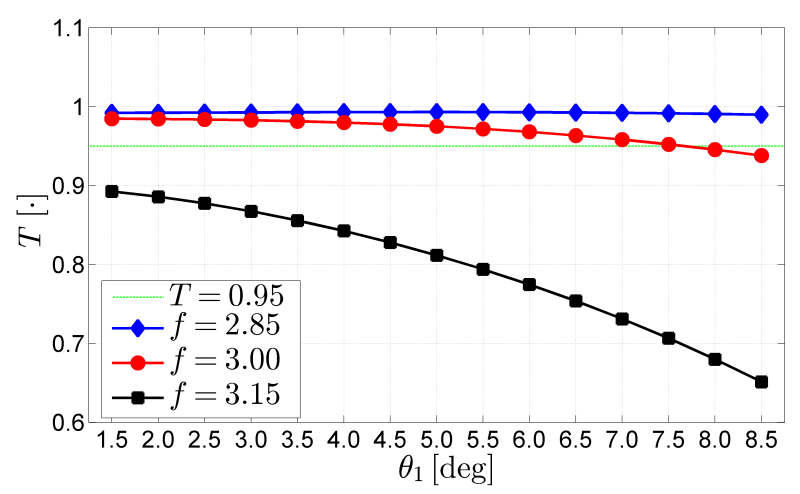

(i)

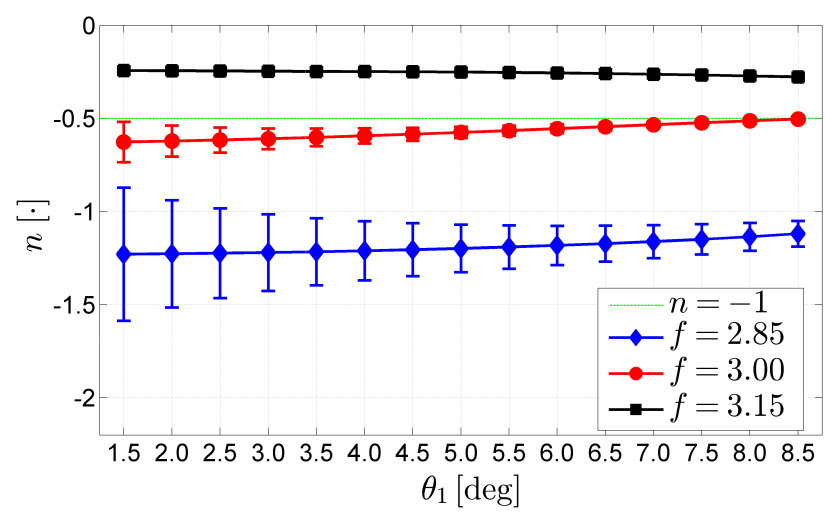

(ii)

Fig. 18: (color online) (i) Transmittance and (ii) refractive index, including error bars denoting the uncertainty in $n$ stemming from the uncertainty in $\theta_{2}$,fit, for MM slab consisting of $22 \times 12$ unit cells containing the optimized design shown in Fig. 17ii as a function of incident angle, $\theta_{1}$, for different frequencies.

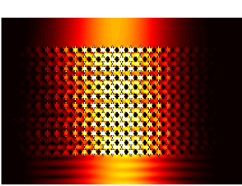

(i)

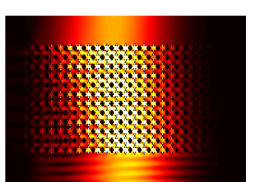

(ii)

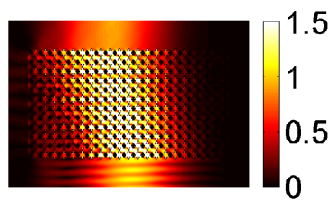

(iii)
Fig. 19: (color online) Amplitude of the solution, $|\psi|$, to the model problem (1)- 3 , in $\boldsymbol{\Omega}_{\mathrm{T}}$ containing a MM slab of $22 \times 12$ unit cells consisting of the optimized design in Fig. 17ii for three different incident angles $\theta_{1}$ at the target frequency $f=3.0$. (i) $\theta_{1}=2.5^{\circ}$ (ii) $\theta_{1}=5.0^{\circ}$ (iii) $\theta_{1}=7.5^{\circ}$.

\section{Conclusions}

A topology optimization based approach for designing meta material slabs exhibiting a prescribed negative refraction under illumination of a plane wave at a specified angle of incidence and target frequency has been proposed. Examples for varying refractive index, angle of incidence and frequency demonstrated the method. An MM slab exhibiting the targeted negative refraction to within $\approx 10 \%$ error with high transmittance, $T \approx 0.98$ for the targeted angle of incidence and frequency was presented. It was shown to be possible to increase the transmittance and reduce the variation in $n$ with incoming angle by taking multiple angles into account in the design process. It was also shown to be possible to improve the transmittance and reduce the variation of $n$ across a band of frequencies by optimizing for multiple frequencies simultaneously. The influence of uniform spatial variations of a design optimized for $\theta_{1} \in\left\{-5^{\circ},-10^{\circ},-15^{\circ}\right\}$ was investigated. While the variations were found to have an impact on the designs performance it was also found that the index of refraction remained negative and the transmittance above $\approx 0.8$ for $\theta_{1} \in\left[-15^{\circ}, 15^{\circ}\right]$ for both the eroded and dilated versions of the design.

The presented approach has the clear benefit that no assumptions that reduce the physical model are imposed, such as applying homogenization, assuming infinite periodicity of the MM, assuming a given boundary condition at the MM interface or considering only the dominant mode inside the MM. On the contrary, the method inherently accounts for edge effects and the more advanced behavior of the field inside the MM. Thus it is expected that any manufactured MMs, designed using the presented approach, should directly function as predicted by the simulation results.

Although the paper treats the acoustic case, the method is directly applicable to both electromagnetic and elastic wave problems by adjusting the relevant material parameters, $\alpha$ and $\gamma$, the wave speed, $c$, and the spatial dimensions. As the contrast in material parameters can be orders of magnitude smaller for both electromagnetic and elastic shear waves, it is the authors' experience that the optimization problem is less challenging in those cases.

\section{Acknowledgments}

The work was financially supported by Villum Fonden through the research project Topology Optimization - the Next Generation (NextOpt).

\section{References}

Andkjær J, Sigmund O (2013) Topology optimized cloak for airborn sound. Journal of Vibration and Acoustics 135:041,011-1 - 041,001-5

Bendsøe MP, Sigmund O (2003) Topology Optimization. Springer

Christiansen RE, Fernandez-Grande E, Sigmund O (2015a) Experimental validation of topology optimized acoustic 
cavity. Journal of the Acoustical Society of America 138 (6):3470-3474

Christiansen RE, Lazarov BS, Jensen JS, Sigmund O (2015b) Creating geometrically robust designs for highly sensitive problems using topology optimization - acoustic cavity design. Structural and Multidiciplinary Optimization 52:737-754 DOI 10.1007/s00,158-015-1265-5

Craster RV, Guenneau S (2013) Acoustic Metamaterials - Negative Refraction, Imaging, Lensing and Cloaking. Springer Science+Business Media Dordrecht

Desmet W (1998) A wave based prediction technique for coupled vibro-acoustic analysis. $\mathrm{PhD}$ thesis, Katholieke Universiteit Leuven

Dühring MB, Jensen JS, Sigmund O (2008) Acoustic design by topology optimization. Journal of Sound and Vibration 317:557-575

Diaz AR, Sigmund O (2009) A toplogy optimization method for design of negative permeability metamaterials. Structural and Multidiciplinary Optimization 41:163-177

Goo S, Kook J, Koo K, Hyun J, Wang S (2014) Acoustic topology optimization for interior acoustic problem using the hybrid finite element - wave based method. In: The Eighth China-Japan-Korea Joint Symposium on Optimization of Structural and Mechanical Systems

Guest JK, Prévost JH, Belytschko T (2004) Achieving minimum length scale in topology optimization using nodal design variables and projection functions. International Journal for Numerical Methods in Engineering 61:238254

Jansen M, Lazarov B, Schevenels M, Sigmund O (2013) On the similarities between micro/nano lithography and topology optimization projection methods. Structural and Multidisciplinary Optimization 48:717-730

Krowne CM, Zhang Y (2007) Physics of Negative Refraction and Negative Index Material - Optical and Electronic Aspects and Diversified Approaches. SpringerVerlag Berlin Heidelberg

Lu L, Yamamoto T, Otomori M, Yamada T, Kazuhiro, Nishiwaki S (2013) Topology optimization of an acoustic metamaterial with negative bulk modulus using local resonance. Finite Elements in Analysis and Design 72:112

Mills EM, Banks ML, Sprague JE, Finkel T (2003) Imaging by flat lens using negative refraction. Nature 426:404

Mortensen NA, Yan M, Sigmund O, Breinbjerg O (2010) On the unambiguous determination of effeffect optical properties of periodic metamaterials: a one-dimensional case study. Journal of the European Optical Society 5:-

Park JH, Ma PS, Kim YY (2015) Design of phononic crystals for self-colimation of elastic waves using topology optimization method. Structural and Multidiciplinary Optimization 51:1199-1209
Pendry JB (2000) Negative refraction makes a perfect lens. Physical Review Letters 85:3966-3969

Philippe FD, Murray TW, Prada C (2015) Focusing on plates: Controlling guided waves using negative refraction. SCIENTIFIC REPORTS 5:11,112 - 1 $11,112-4$

Pluymers B (2006) Wave based modelling methods for steady-state vibro-acoustics. PhD thesis, Katholieke Universiteit Leuven

Svanberg K (1987) The method of moving asymptotes a new method for structural optimization. International Journal for Numerical Methods in Engineering 24:359373

Svanberg K (2002) A class of globally convergent optimization methods based on conservative convex seperable approximations. SIAM Journal of Optimization 12:555573

Veselago VG (1968) The electrodynamics of substances with negative values of $\epsilon$ and $\mu$. Soviet Physics Uspekhi 10:509-514

Wang F, Lazarov BS, Sigmund O (2011) On projection methods, convergence and robust formulations in topology optimization. Structural Multidiciplinary Optimization 43:767-784

Xu S, Cai Y, Cheng G (2010) Volume preserving nonlinear density filter based on heaviside projections. Structural and Multidiciplinary Optimization 41:495-505

Xu T, Agrawal A, Abashin M, Chau KJ, Lezec HJ (2013) All-angle negative refraction and active flat lensing of ultraviolet light. Nature 497:470-474

Zhang S, Fan W, Panoiu NC, Malloy KJ, Osgood RM, Brueck SRJ (2005) Experimental demonstration of nearinfrared negative-index metamaterials. Physical Review Letters 95:137,404-1 - 137,404-4

Zhang S, Yin L, Fang N (2009) Focusing ultrasound with an acoustic metamaterial network. Physical Review Letters 102:194,301-1 - 194,301-4

Zhang X, Liu Z (2004) Negative refraction of acoustic waves in two-dimensionl phononic crystals. Applied Physics Letters 85:341-343

Zhou M, Lazarov B, Sigmund O (2016) Topology optimization for optical microlithography with partially coherent illumination. Submitted

Zhou S, Li W, Sun G, Li Q (2010) A level-set procedure for the design of electromagnetic metamaterials. Optical Express 18 